\title{
Recovery of a critically ill patient with COVID-19 myocarditis
}

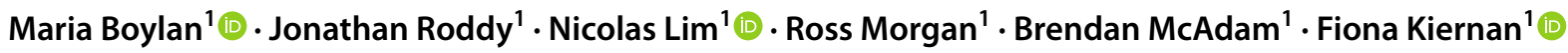

Received: 3 June 2021 / Accepted: 9 June 2021 / Published online: 16 June 2021

(c) Royal Academy of Medicine in Ireland 2021

\begin{abstract}
Myocarditis is a concerning potential consequence of COVID-19 infection, attributed to ventricular dysfunction, cardiac fibrosis, ventricular arrhythmias, cardiogenic shock, and sudden cardiac death. Recently, the Israeli Health Ministry announced that a small number of cases of myocarditis may be linked to second dose of Pfizer's BioNTech-partnered COVID-19 vaccine. The long-term impact of COVID-19 myocarditis and coronary microthrombosis which has also been described and the best therapies for these complications remain unknown. Indeed, monomorphic ventricular tachycardia and regular ventricular arrhythmias have previously been found to be more common in those recovered from myocarditis than in acute myocarditis itself. Follow-up assessment of cardiac function has been suggested for this cohort to detect and possibly prevent further cardiac events in the rehabilitation phase. Functional capacity has been shown to be a better determinant of long-term morbidity than diagnostic testing alone, but integrated approach is likely the way forward in clinical follow-up. Assessment of residual complications in the post-COVID-19 recovery phase may identify the population burden of long-term cardiac disease as a direct consequence of COVID-19.
\end{abstract}

Keywords Cardiac function · Corticosteroids · COVID-19 myocarditis · Critical care · Outcomes $\cdot$ Troponin

\section{Dear Editor,}

Myocarditis is a concerning potential consequence of COVID-19 infection, attributed to ventricular dysfunction, cardiac fibrosis, ventricular arrhythmias, cardiogenic shock, and sudden cardiac death [1]. Recently, the Israeli Health Ministry announced that a small number of cases of myocarditis may be linked to second dose of Pfizer's BioNTechpartnered COVID-19 vaccine, with the majority discovered in men between 16 and 30 years. In their unpublished report, the Israeli Health Officials declared 27 cases of myocarditis were discovered following the first dose, out of a total of $5,401,150$ vaccinated individuals. One hundred and twentyone cases out of a total of 5,049,424 vaccinated individuals within 30 days of second dose were also revealed [2].

The long-term impact of COVID-19 myocarditis and coronary microthrombosis, which has also been described, and the best therapies for these complications remain unknown. Indeed, monomorphic ventricular tachycardia and regular ventricular arrhythmias have previously been found to be

Maria Boylan

mariabboylan@yahoo.co.uk

1 Beaumont Hospital, Beaumont Road, Dublin, Ireland more common in those recovered from myocarditis than in acute myocarditis itself [3]. A follow-up assessment of cardiac function has been suggested for this cohort to detect and possibly prevent further cardiac events in the rehabilitation phase [4]. Functional capacity has been shown to be a better determinant of long-term morbidity than diagnostic testing alone, but integrated approach is likely the way forward in clinical follow-up [5]. Assessment of residual complications in the post-COVID-19 recovery phase may identify the population burden of long-term cardiac disease as a direct consequence of COVID-19. This case-based review describes a patient who required critical care treatment for COVID-19 pneumonitis and multi-organ failure. He was diagnosed with myocarditis during this critical period and is currently under surveillance in the COVID-19 ICU Survivorship Clinic. This letter should provide reassurance that this group may return towards their baseline in the medium term.

A 61-year-old gentleman was admitted to our intensive care unit (ICU) with coronavirus (COVID-19) pneumonitis during the first wave of severe acute respiratory syndrome coronavirus 2 (SARS-CoV-2). His past medical history was significant for multiple cardiovascular risk factors such as hypertension, type 2 diabetes mellitus, and hyperlipidaemia; however, he had no history of previous myocardial 
Fig. 1 Laboratory values of troponin T (ng/L) graph illustrating daily rise in troponin $\mathrm{T}$ levels. Troponin level peaked at $6142 \mathrm{ng} / \mathrm{L}$ on day 35 in intensive care. Troponin T levels began to normalise following administration of methylprednisolone

\section{Laboratory Values}

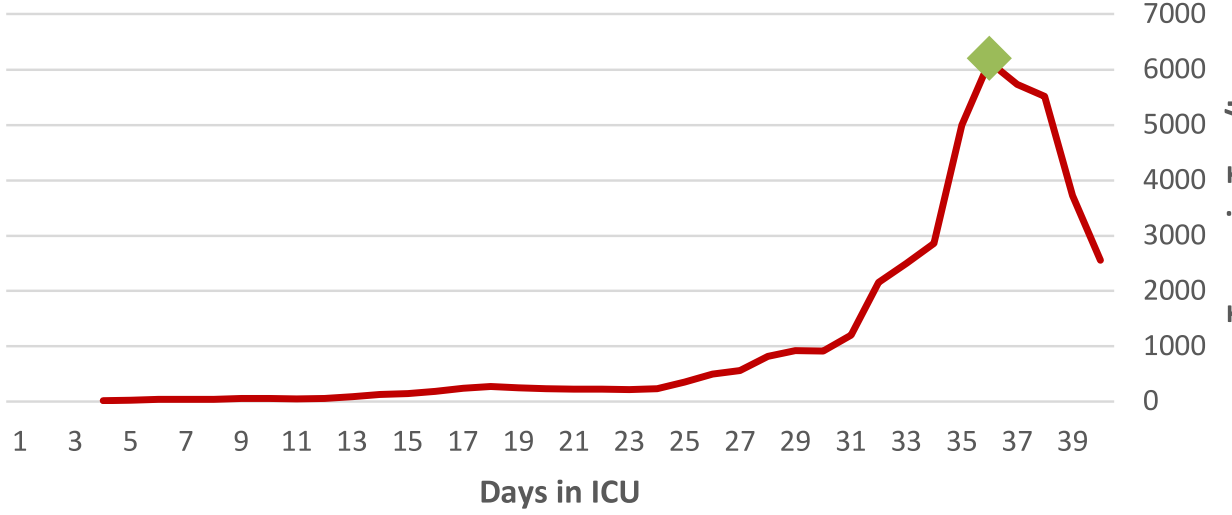

5000

4000

3000

2000

000

Days in ICU infarction. Additional medical background included asthma, chronic obstructive pulmonary disease, hypothyroidism, and rectal cancer requiring anterior resection in 2017.

Following a trial of high flow oxygen via nasal cannulae, he was intubated and ventilated for refractory hypoxaemia using lung-protective ventilation and prone positioning. He developed multiorgan failure with hypotension requiring vasopressor support. He developed acute renal failure, which ultimately required renal replacement therapy. His renal function subsequently recovered prior to discharge from ICU. Routine monitoring detected an increase in troponin $\mathrm{T}(\mathrm{TnT}) 3$ weeks after admission to ICU, and 5 weeks after his diagnosis with SARS-Cov-2. TnT peaked at $6142 \mathrm{ng} / \mathrm{L}$ following increase over four sequential days, as shown in Fig. 1. Pro-NT brain natriuretic peptide (BNP) serum level taken at that time was elevated at $2180 \mathrm{pg} / \mathrm{ml}$. Differential diagnosis included acute coronary syndrome, sepsis-related cardiomyopathy, and stress-induced cardiomyopathy. There was no evidence of cardiac ischaemia or arrhythmia on electrocardiogram (ECG), and echocardiography depicted normal biventricular function with no regional wall motion abnormality, no significant valve abnormality, and no pericardial effusion. Cardiac angiography found no evidence of coronary artery disease.

A definitive diagnosis of myocarditis could not be made without cardiac magnetic resonance (CMR) or endomyocardial biopsy, neither of which was appropriate for the patient at this time. A presumptive diagnosis of COVID-19 myocarditis was made in view of this day-onday increase in cardiac enzymes, which was consistent with available literature, and pulsed methylprednisolone was commenced ${ }^{1}$. Cardiac enzyme levels improved after induction of steroids (Fig. 1); his ventilation was weaned, and he was discharged to the ward. A subsequent CMR performed at 3 months post-ICU discharge confirmed the diagnosis of myocarditis with fibrosis on the late gadolinium enhancement (LGE) sequences observed in midwall and subepicardial aspect of the basal segments of the septum, inferior septum, inferior and lateral walls. There was no evidence of myocardial oedema on the T2 STIR images and T2 maps. There was no evidence of subendocardial infarction on the late gadolinium enhancement sequence (Fig. 2).

He was discharged to the community and reviewed in the post-intensive care clinic at 6 months where he denied any cardiac symptoms relating to chest pain or dyspnoea. BNP was $<50 \mathrm{pg} / \mathrm{ml}$, and a repeat echocardiogram showed normal ventricular function with an ejection fraction of 55\%. Pulmonary function tests showed a reduced FEV1/FVC at $68 \%$ and diffusion capacity of $55 \%$ predicted. There was evidence of resolution of COVID19 pneumonitis on computed tomography (CT) thorax, although some peribronchovascular nodularity remained. He managed $380 \mathrm{~m}$ on 6-min walk test (6MWT) without desaturation, and 11 repetitions in the 30-s sit-to-stand test, demonstrating both good muscle strength and functional capacity. Grip strength was $27 \mathrm{~kg}$ on right, and $30 \mathrm{~kg}$ on left (right hand dominant). His self-assessed health status was in the second highest level of health, 'very good', which is higher than expected for his age and gender. Table 1 shows scores for the eight scales of SF36. On individual item questioning, he reported 'some' limitations in terms of strenuous activities, but 'no' limitations for moderate activities. He has not yet returned to work.

1 This was prior to the publication of the recovery trial. 
Fig. 2 Cardiac MRI - shortaxis slices showing late gadolinium enhancement (LGE). Left ventricle is normal in size and volume with normal wall thickness and normal systolic function (LVEF 59\%). There is no evidence of prior infarction on the LGE, but there are small foci of mild wall fibrosis in the basal segments of the septum, inferior septum, inferior and lateral walls and in the subepicardium of the basal anterio-lateral and lateral walls. This is consistent with prior myocarditis. There is non-specific mid-wall fibrosis at the basal superior and basal mid inferior RV-LV insertion points. Findings are consistent with prior myocarditis with normal LVEF

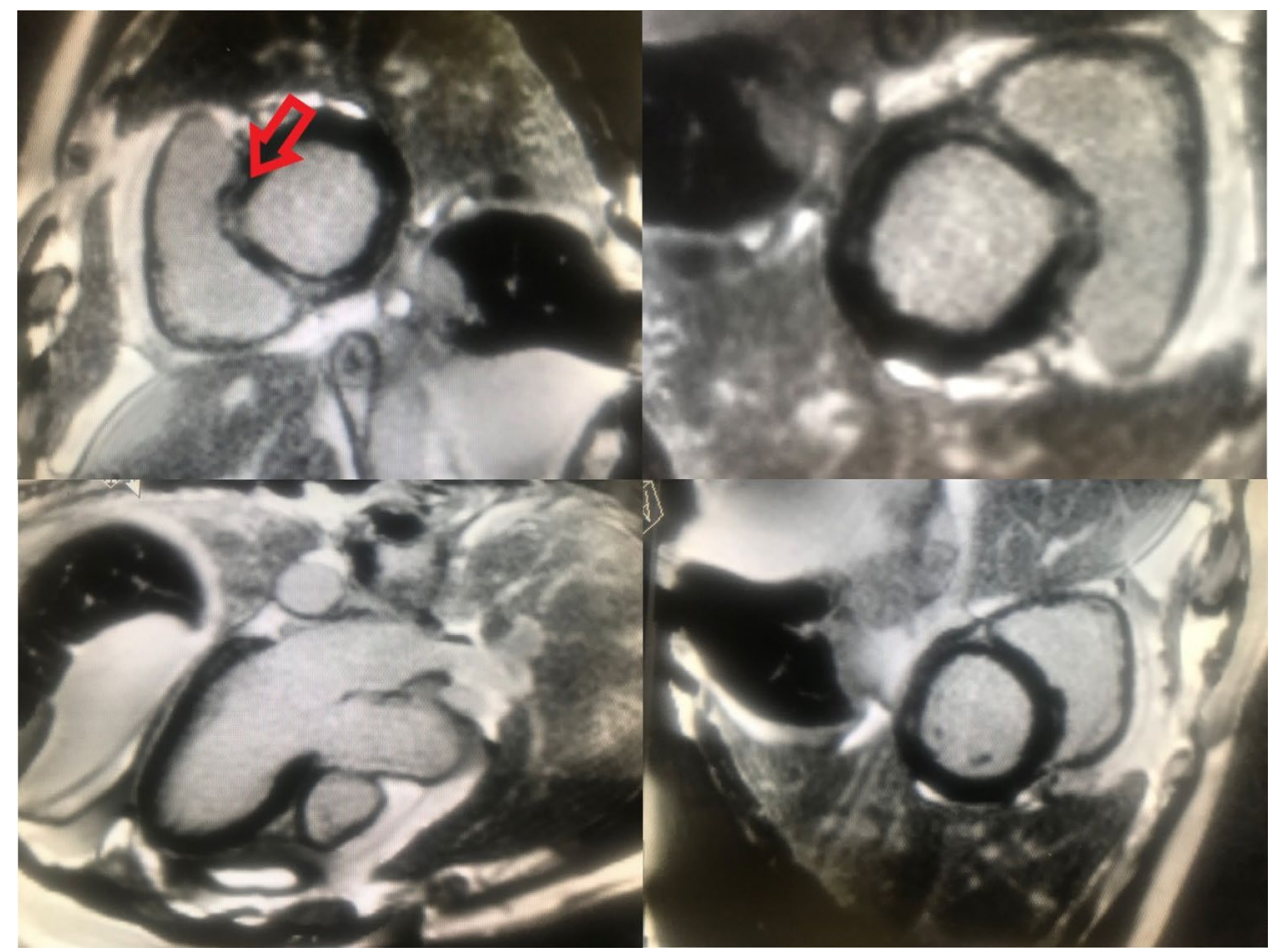

\section{Discussion}

Myocarditis has been recognised as a cause of death in patients diagnosed with COVID-19 [6]. It is characterised by focal inflammation within the myocardium, with a subsequent risk of arrhythmias, fulminant heart failure, and cardiogenic shock $[1,7]$. Putative pathophysiological mechanisms behind COVID-19 myocarditis include the cytokine storm induced by an imbalance between $\mathrm{T}$ helper-1 and $\mathrm{T}$ helper- 2 cells with resulting cytokinemediated cardiotoxicity, imbalance in the angiotensin converted enzyme (ACE)-angiotensin axis, direct viral myocardial and endocardial invasion-mediated via ACE tropism [8]. An additional mechanism of cardiac injury has been described by Pellegrini et al., from the Italian autopsy series. Cardiac myocyte necrosis from microthrombi was

Table 1 Eight domains of SF 36

\begin{tabular}{ll}
\hline & Scores (/100) \\
\hline Physical functioning & 95 \\
Role limitation physical & 100 \\
Role limitation emotional & 100 \\
Energy & 95 \\
Emotional well-being & 100 \\
Social functioning & 100 \\
Pain & 70 \\
General health & 95 \\
\hline
\end{tabular}

different in composition from thrombi from catheter aspirates from patients with acute coronary syndrome [9].

Much has been learned regarding this novel viral myocarditis since January 2020, and while the European Society of Cardiology (ESC) and American Heart Association (AHA) recommend endomyocardial biopsy as the definitive method for diagnosis in myocarditis, there is a risk of false-negatives, as the disease can present in a focal manner [10, 11]. More recently, CMR has been suggested as an accurate diagnostic tool $[12,13]$. Importantly, there are increasing reports of significant CMR changes in patients with COVID-19, despite clinically mild symptoms [14]. Based on these findings, it is now suggested that even patients with mild myocarditis should continue to be followed in the medium term, in particular in patients who had elevated cardiac enzymes and BNP [4]. Due to the novel nature of the disease, the literature is, of course, lacking in terms of medium-term and long-term outcomes of COVID-19 related myocarditis.

Prior literature from non-COVID-19 viral myocarditis suggests that the rate of sudden cardiac death or lifethreatening ventricular arrhythmias may be as high as $6.6 \%$ [15]. While CMR with LGE, post-infection, could identify the at-risk group [3], functional capacity has been shown to be both independently and incrementally associated with prognosis in patients with cardiac disease and is a better predictor of outcome than diagnostic testing alone [16]. The late risk of arrhythmias in these patients is currently unknown and perhaps Holter or telemetry monitoring during rehab may be prudent. 
Assessment of functional capacity is particularly important for patients who have required critical care for multiorgan failure either owing to, or concurrent with, their diagnosis of myocarditis. A relatively straightforward assessment of functional capacity can be performed in the outpatient clinic. While cardiopulmonary exercise testing remains the gold standard for prognostic stratification for long-term patients, the 6MWT can provide similar information [17]. The American Thoracic Society (ATS) has recommended 6MWT as a measure of functional status, and for prognostication in patients with respiratory disease and cardiac failure [18]. Maximal distance walked during the 6MWT is an indicator of submaximal exercise tolerance, and therefore similar to activity of daily living. A maximal distance of $<300 \mathrm{~m}$ is predictive of morbidity in patients with mild-to-moderate heart failure [19].

Our patient managed $380 \mathrm{~m}$, however, his functional limitations were also limited by his post-ICU status and diagnosis of ARDS. A recent systematic review of the 6MWT in ICU survivors found that the pooled mean distance was $360 \mathrm{~m}$ at 3 months post-ICU discharge, increasing to 395 $\mathrm{m}$ at 6 months [20]. A previous small prospective cohort study of 56 patients found that median 6MWT distance was $392 \mathrm{~m}$ [21]. Despite assumptions that ICU-related myopathy may be a driver of reduced distance, well-recognised risk factors such as severity of illness, use of steroids, and neuromuscular blockers have not been found to be associated with maximal distance achieved [20]. Therefore, the 6MWT is predominantly useful in assessing functional outcomes related to respiratory and cardiac disease in ICU patients.

The grip strength and sit-to-stand tests are a further reliable measure of functional capacity and muscle strength [22, 23]. Mean grip strength was shown to be $29.3 \mathrm{~kg}$ on the right and $27.3 \mathrm{~kg}$ on the left for cardiac patients already engaged in a rehab program [24]. Median grip strength in ICU survivors at 3 months was $20.4 \mathrm{~kg}$ [21]. Our patient's grip strength was above these scores.

While there are few papers examining the sit-to-stand test for ICU survivors, a quasi-example is the GymNAST study which found that recovery of speed to stand (as opposed to repetitions) returned at 2 months post-ICU discharge for those with ICU-acquired muscle weakness [25].

The majority of papers examining SF36 scores in the cardiac literature relate to congestive heart failure, postmyocardial infarction, and post-operative states, with no real evidence on quality of life scores for viral myocarditis. However, the health outcomes research literature does examine the eight domains of the SF36 for ICU patients. One particularly relevant paper found that SF36 scores were higher for Middle East Respiratory Syndrome (MERS) survivors than non-MERS ICU survivors [26].
Although there is an assumption that ICU survivors have poorer quality of life scores post-discharge than age and sex-matched population controls, a cross-sectional study from Sweden found that scores were relatively similar [27]. Those with worse outcomes than the population average were male, single, and on sick leave prior to ICU admission. Our patient had only one of these risk factors (although he has not yet returned to work post-discharge) and his scores are above expected values for all eight domains of the SF-36 survey.

While CMR is a useful tool to measure response to treatment in selected patients, there is no evidence on the optimal time to perform follow-up CMR [28] and there are no established myocarditis specific biomarkers that can predict outcome [29]. BNP has been shown to be associated with long-term outcomes for patients with cardiomyopathy [8] as well as medium- to long-term outcomes after ICU discharge [30]. Since BNP is recognised as an optimal means of guiding treatment in the ambulatory setting for patients with heart failure [31], it is reasonable to consider that this as an additional tool in the follow-up of ICU patients with myocarditis.

\section{Conclusion}

Viral myocarditis in patients can occur with SARS-CoV-2 infection, causing a fulminant cardiomyopathy. The diagnosis of COVID-19 associated myocarditis can be challenging, particularly in the critically ill population. This patient had no arrhythmogenic or anatomical insult. Currently, ESC guidelines do not recommend steroids in mild cases of COVID-19 myocarditis [32]. Aggressive treatment of isolated elevated troponin levels without cardiovascular compromise, in this case, may have prevented the development of chronic myocarditis and subsequent inflammatory cardiomyopathy. This incidental diagnosis highlights the importance of regular serum screening in all COVID-19 patients and the potential merits of early intervention.

As of June 2, 2021, the centre for disease control and prevention continues to advise COVID-19 vaccination for persons aged 12 years and older, taking into account the greater risk of COVID-19 illness possible severe complications.

Since even mild myocarditis is associated with long-term morbidity, it is important that this group of patients is followed in the recovery phase. Outpatient assessment of functional capacity can be used alongside diagnostic testing to detect the at-risk group. It is reassuring that good functional outcomes were observed in our patient who was affected by the double burden of myocarditis and COVID-19 pneumonitis, with associated multi-organ failure. 


\section{Declarations}

Ethical approval This article does not contain any studies with human participants or animals performed by any of the authors.

Consent for publication Written consent was obtained from a patient.

Conflict of interest The authors declare no competing interests.

\section{References}

1. Siripanthong B et al (2020) Recognizing COVID-19-related myocarditis: the possible pathophysiology and proposed guideline for diagnosis and management. Heart Rhythm 17(9):1463-1471

2. Carlson R (2021) Israel investigates heart issues related to PfizerBioNTech COVID-19 Vaccine

3. Peretto $\mathrm{G}$ et al (2020) Ventricular arrhythmias in myocarditis: characterization and relationships with myocardial inflammation. J Am Coll Cardiol 75(9):1046-1057

4. Mitrani RD, Dabas N, Goldberger JJ (2020) COVID-19 cardiac injury: Implications for long-term surveillance and outcomes in survivors. Heart Rhythm 17(11):1984-1990

5. Forman Daniel E et al (2017) Prioritizing functional capacity as a principal end point for therapies oriented to older adults with cardiovascular disease: a scientific statement for healthcare professionals from the American Heart Association. Circulation 135(16):e894-e918

6. Ruan Q et al (2020) Clinical predictors of mortality due to COVID-19 based on an analysis of data of 150 patients from Wuhan, China. Intensive Care Med 846-848

7. Esfandiarei M, McManus BM (2008) Molecular biology and pathogenesis of viral myocarditis. Annu Rev Pathol 3:127-55

8. Akita K, Tsurata H, Yuasa $\mathrm{S}$ et al (2018) Prognostic significance of repeated brain natriuretic peptide measurements after percutaneous transluminal septal myocardial ablation in patients with drug-refractory hypertrophic obstructive cardiomyopathy. Open Heart 5:e000786

9. Pellegrini D et al (2021) Microthrombi as a major cause of cardiac injury in COVID-19. Circulation 143(10):1031-1042

10. Kociol RD et al (2020) Recognition and initial management of fulminant myocarditis: a scientific statement from the American Heart Association. Circulation 141(6):e69-e92

11. Caforio AL et al (2013) Current state of knowledge on aetiology, diagnosis, management, and therapy of myocarditis: a position statement of the European Society of Cardiology Working Group on Myocardial and Pericardial Diseases. Eur Heart J 34(33):263648, 2648a-2648d

12. Ferreira VM et al (2018) Cardiovascular magnetic resonance in nonischemic myocardial inflammation: expert recommendations. J Am Coll Cardiol 72(24):3158-3176

13. Grün $\mathrm{S}$ et al (2012) Long-term follow-up of biopsy-proven viral myocarditis: predictors of mortality and incomplete recovery. J Am Coll Cardiol 59(18):1604-15

14. McKinney J et al (2020) COVID-19-Myocarditis and return to play: reflections and recommendations from a Canadian Working Group. Can J Cardiol

15. Marc-Alexander $\mathrm{O}$ et al (2018) Predictors of long-term outcome in patients with biopsy proven inflammatory cardiomyopathy. $\mathrm{J}$ Geriatr Cardiol 15(5):363-371
16. Tang WH, Topol EJ, Fan Y et al (2014) Prognostic value of estimated functional capacity incremental to cardiac biomarkers in stable cardiac patients. J Am Heart Assoc 3(5):e000960

17. Wegrzynowska-Teodorczyk K, Rudzinska E, Lazorczyk M et al (2013) Distance covered during a six-minute walk test predicts long-term cardiovascular mortality and hospitalisation rates in men with systolic heart failure: an observational study. J Physiother 59(3): 177-187

18. Society, A.T. (2002) ATS Statement: guidelines for the sixminute walk test. Am J Respir Crit Care Med 166:111-117

19. Rostagno C, Olivo G, Comeglio M et al (2003) Prognostic value of 6-minute walk corridor test in patients with mild to moderate heart failure: comparison with other methods of functional evaluation. Eur J Heart Fail 5(3):247-252

20. Parry SM, Nalamalapu SR, Nunna K et al (2021) Six-minute walk distance after critical illness: a systematic review and meta-analysis. J Intensive Care Med 36(3):343-351

21. Solverson KJ, Grant C, Doig CJ (2016) Assessment and predictors of physical functioning post-hospital discharge in survivors of critical illness. Ann Intensive Care 6(1):92

22. Ozalevli S et al (2007) Comparison of the sit-to-stand Test with 6 min walk test in patients with chronic obstructive pulmonary disease. Respir Med 101(2):286-93

23. Jones SE et al (2013) The five-repetition sit-to-stand test as a functional outcome measure in COPD. Thorax 68(11):1015-1020

24. Puthoff ML, Saskowski D (2013) Reliability and responsiveness of gait speed, five times sit to stand, and hand grip strength for patients in cardiac rehabilitation. Cardiopulm Phys Ther J 24(1):31-37

25. Thomas $\mathrm{S}$ et al (2016) Recovery of sit-to-stand function in patients with intensive-care-unit-acquired muscle weakness: results from the general weakness syndrome therapy cohort study. J Rehabil Med 48(9):793-798

26. Batawi $\mathrm{S}$ et al (2019) Quality of life reported by survivors after hospitalization for Middle East respiratory syndrome (MERS). Health Qual Life Outcomes 17(1):101

27. Orwelius L, Fredrikson M, Kristenson M et al (2013) Critical care (London, England), 17(5):R236, Health-related quality of life scores after intensive care are almost equal to those of the normal population: a multicenter observational study. Crit Care 17(5):R236. https://doi.org/10.1186/cc13059

28. Bohnen S, Radunski UK, Lund GK et al (2017) Tissue characterization by $\mathrm{T} 1$ and $\mathrm{T} 2$ mapping cardiovascular magnetic resonance imaging to monitor myocardial inflammation in healing myocarditis. Eur Heart J Cardiovasc Imaging 18(7):744-751

29. Tschope C, Ammirati E, Bozkurt B et al (2020) Myocarditis and inflammatory cardiomyopathy: current evidence and future directions. Nat Rev Cardiol. https://doi.org/10.1038/s41569020-00435-x

30. Gayat E, Cariou A, Deye N et al (2018) Determinants of long-term outcome in ICU survivors: results from the FROG-ICU study. Crit Care 22(8). https://doi.org/10.1186/s13054-017-1922-8

31. Bhardwaj A, Rehman SU, Mohammed AA et al (2012) Quality of life and chronic heart failure therapy guided by natriuretic peptides: results from the ProBNP Outpatient Tailored Chronic Heart Failure Therapy (PROTECT) study. Am Heart J 164(5):793-799

32. Ammirati E et al (2020) Management of acute myocarditis and chronic inflammatory cardiomyopathy: an expert consensus document. Circulation. Heart Fail 13(11):e007405-e007405

Publisher's Note Springer Nature remains neutral with regard to jurisdictional claims in published maps and institutional affiliations. 